\title{
Temporally flat top pulse generation from gain switched semiconductor lasers based on a polarization interferometer with variable transfer function
}

\author{
Antonio Consoli , Ignacio Esquivias
}

\begin{abstract}
A B S T R A C T
We propose the use of a polarization based interferometer with variable transfer function for the generation of temporally flat top pulses from gain switched single mode semiconductor lasers. The main advantage of the presented technique is its flexibility in terms of input pulse characteristics, as pulse duration, spectral bandwidth and operating wavelength. Theoretical predictions and experimental demonstrations are presented and the proposed technique is applied to two different semiconductor laser sources emitting in the $1550 \mathrm{~nm}$ region. Flat top pulses are successfully obtained with input seed pulses with duration ranging from 40 ps to $100 \mathrm{ps}$.
\end{abstract}

\section{Introduction}

Optical pulses with duration of tens of picoseconds find application in a wide variety of fields: optical communication channels, imaging, material processing, sensing, optical scanning and ranging [1]. Pulse temporal profile is often relevant, as specific applications require the use of particular pulse shapes. This is the case of flat top pulses which are employed in a large variety of applications, e.g. retiming of high data bit rate optical communication channels in $C$ band [2], efficient pumping of free electron lasers in the deep Ultra Violet wavelength region [3] and amplitude and phase characterization of ultra-short pulses [4].

Common methods for flat top pulse generation are based on spectral filtering of Gaussian, transform limited, ultra-short pulses obtained from mode locked lasers [5]. The general approach is based on pulse spectral filtering by means of a specifically designed photonic device, adapted to the intensity and phase profiles of the seed pulse. The required photonic filter can be implemented with interferometers [6-8], Fiber Bragg Gratings (FBGs) [9-11] or optical differentiators [12,13].

Usually there is a compromise between device design, implementation and tunability. All fiber implementation is usually designed only for a specific input pulse, which has been previously carefully characterized. Free optics elements and movable parts allows the modification of the photonic filter Transfer Function (TF) and tunability of the device in terms e.g. of obtainable output pulse profiles, operating wavelength and input pulses. The most general case of arbitrary pulse shaping uses a pair of diffraction gratings and a programmable spatial mask: the spectral content is dispersed, properly filtered and then recombined [14]. This solution is widely flexible, but bulky. More recent works have reported fiber implementation and some degree of freedom on its TF given by mechanical action on the fiber. For example, in [7] the Extinction Ratio (ER) of a dual mode fiber interferometer is tuned by twisting the dual mode fiber. In a similar way, in [12] the spectral transmission dip of the proposed differentiator is varied by applying axial straining to the Long Period Fiber Grating (LPFG). However, apart from the ER tuning, the spectral characteristics of the filter, e.g. the Free Spectral Range (FSR) of the interferometer or the central frequency of the differentiator, cannot be varied, thus fixing the operating wavelength and limiting the spectral characteristics of the allowed input pulses.

We have previously proposed a hybrid fiber and free space optics implementation of a polarization based 50:50 Mach-Zehnder (MZ) interferometer with variable delay, for pulse shortening of gain-switched (GS) single mode semiconductor lasers [15]. The spectral TF of the proposed device was properly tuned for suppressing the red frequencies side of the pulse spectrum, thus reducing the falling edge of the pulse temporal profile and its total duration. In [15] pulse shortening has been demonstrated with a Distribute Feedback (DFB) laser and a Vertical Cavity Surface 
Emitting Laser (VCSEL), both emitting in the $1550 \mathrm{~nm}$ region. The pulse at the interferometer input had durations in the range of tens of picoseconds, typical of GS lasers, similar to the pulses presented in this work.

In this manuscript, we extend our previous work with the implementation of a polarization based interferometer, similar to the one presented in [15], in which the input coupling, output coupling and temporal delay between the two arms can be easily tuned. We demonstrate flat top pulse generation from a DFB laser and a VCSEL operating at different wavelengths in the $1550 \mathrm{~nm}$ region. Different pulses were generated from the GS devices by varying the gain switching parameters and flat top pulses are obtained in all cases by properly tuning the interferometer parameters.

This work is organized as follows: the principle of operation of the proposed technique and the performed numerical simulations are presented in Section 2; Section 3 describes the experimental results and in Section 4, the main conclusions are presented.

\section{Principle of operation and numerical simulations}

The principle of operation of the proposed technique is based on the use of a chirped pulse at the interferometer input. Pulse shaping is obtained with spectral amplitude filtering, based on the time-frequency correspondence of the input chirped pulse. The central part of the pulse spectrum, which corresponds to the pulse peak in time, is properly attenuated in order to flattens the pulse peak in time and obtain a flat top temporal profile at the interferometer output. This corresponds to use a notch-like filter, with minimum of transmission placed at the pulse spectrum central frequency and with suitable notch depth.

The proposed notch filter is an interferometer with variable TF, in which the ER can be changed by varying the coupling ratios between the two interferometer arms $\left(k_{1}\right.$ and $\left.k_{2}\right)$ and its FSR can be tuned by varying the temporal delay $\tau$ between the interferometer arms. Experimentally, this solution is implemented with a polarization based interferometer, described in detail in Section 3.

Pulses at the interferometer input are generated by gain switching a semiconductor laser, which gives almost linearly chirped pulses. The entire system has been numerically simulated. Pulses at the interferometer input have been obtained in simulations using the density rate equations for photons, carriers and phase [15] with typical parameters for a $1550 \mathrm{~nm}$ DFB laser [16]. Pulses are generated by gain switching the laser with the current $I(t)$, given by the sum of a Direct Current (DC) term, $I_{\text {BIAS, and a }}$ sinusoidal current with amplitude $I_{\mathrm{AMP}}$ and frequency $f_{\mathrm{GS}}$, i.e. $I(t)=$ $I_{\mathrm{BIAS}}+I_{\mathrm{AMP}} \cdot \sin \left(2 \pi f_{\mathrm{GS}} t\right)$.

The output pulse power, $P(t)$, and phase, $\phi(t)$, are obtained from the density rate equations and the pulse complex envelope is calculated as $E(t)=\sqrt{P(t)} \exp [j \phi(t)]$, where $t$ is the time variable

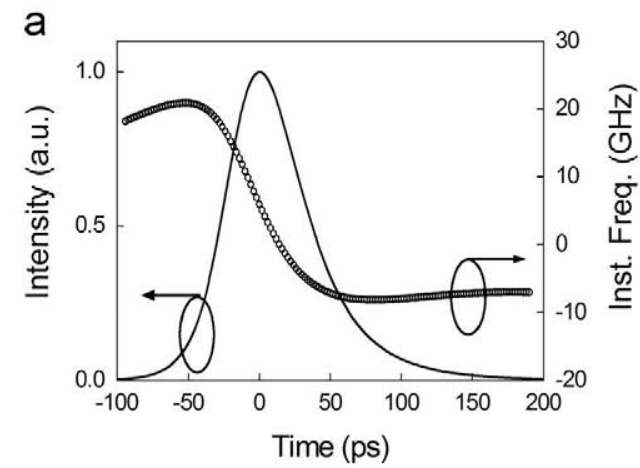

and $j$ is the imaginary unit. The instantaneous frequency $\nu(t)$ is calculated from the time derivative of the phase, i.e. $\nu(t)=(2 \pi)^{-1} d$ $(\phi(t)) / d t$.

The complex pulse spectrum is then obtained as $\tilde{E}(f)=F T[E(t)]$, where $F T$ is the Fourier Transform operator and $f$ the frequency domain variable.

Fig. 1 show the simulation results for $I_{\mathrm{BIAS}}=I_{\mathrm{TH}}, I_{\mathrm{AMP}}=4 I_{\mathrm{TH}}$ and $f_{\mathrm{GS}}=1 \mathrm{GHz}$, where $I_{\mathrm{TH}}$ is the threshold current of the simulated laser. The gain switching parameters ( $I_{\mathrm{BIAS}}, I_{\mathrm{AMP}}$ and $\left.f_{\mathrm{GS}}\right)$ have been chosen in order to have similar results in comparison with experiments. The simulated pulses have Full Width Half Maximum (FWHM) duration of about $65 \mathrm{ps}$ and a spectral bandwidth at $1 / e^{2}$ of $33.5 \mathrm{GHz}$.

Typical features of pulses from GS semiconductor lasers can be observed in Fig. 1(a): asymmetric temporal intensity profile and carrier induced negative variation of instantaneous frequency. In Fig. 1(b), the expected broadened spectrum, with intensity modulation due to the relaxation oscillations at this bias point, and the corresponding group delay are shown.

The interferometer TF, $H(f)$, of a MZ interferometer has been numerically implemented as $H(f)=j \sqrt{k_{1}\left(1-k_{2}\right)}+j \sqrt{k_{2}\left(1-k_{1}\right)}$ $\exp (j 2 \pi f \tau)$ [17] where $\tau$ is the temporal delay between the two interferometer arms and $k_{1}$ and $k_{2}$ are the coupling ratios at the input and output coupler, respectively. For each optical coupler, the coupling ratio $k$ is defined as the fraction of output power of the output port in direct pass to the total output power. The pulse spectrum at the interferometer output is calculated as $\tilde{E}_{\text {OUT }}(f)=$ $\tilde{E}(f) H(f)$ and its temporal complex envelope, $E_{\text {OUT }}(t)$, is obtained after Inverse Fourier Transforming (IFT), as $E_{\text {OUT }}(t)=\operatorname{IFT}\left[\tilde{E}_{\text {OUT }}(f)\right]$.

In order to compare the simulated pulses at the interferometer output with an ideal rectangular pulse, we introduce a simple Flat Top Form Factor (FTFF), defined as:

FTFF $=\frac{\int_{t 1}^{t 2}\left[y(t)-y_{\mathrm{TH}}\right] d t}{\int_{t 1}^{t 2}\left[\operatorname{rect}(t)-y_{\mathrm{TH}}\right] d t}$

where $y(t)$ is the pulse under study, rect $(t)$ is an ideal rectangular pulse, with constant width given by $W=t_{2}-t_{1}$, and $t_{1}$ and $t_{2}$ are the time instants in which $y(t)$ crosses the threshold value $y_{\mathrm{TH}}$. The FTFF definition is schematically depicted in Fig. 2.

The FTFF gives an estimation of how much the waveform under study resembles an ideal rectangular pulse with a given width, set by the threshold value $y_{\mathrm{TH}}$. If $y(t)$ is an ideal rectangular pulse, $\mathrm{FTFF}=1$, in any other case, FTFF $<1$ and its value depends on the shape of $y(t)$ and $y_{\mathrm{TH}}$. We set $y_{\mathrm{TH}}$ equal to $1 / e^{2}$ of the pulse maximum, which gives $\mathrm{FTFF}=0.48$, for the simulated pulse with asymmetric intensity profile shown in Fig. 1.

We performed simulations with the aim of maximizing the FTFF of the output pulse, using at the interferometer input the

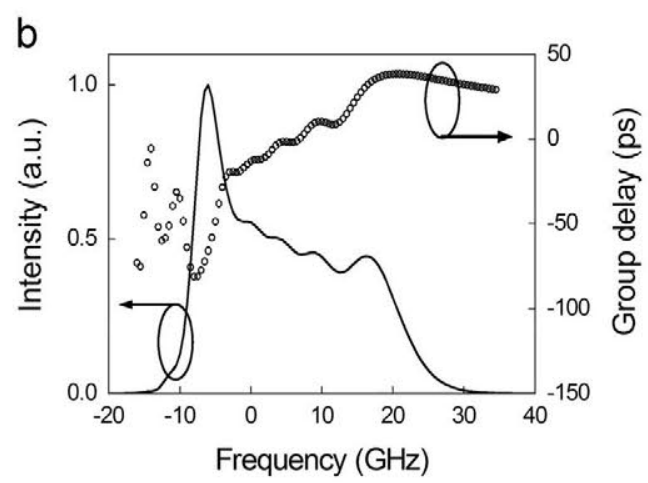

Fig. 1. Simulation results for the simulated GS DFB laser with $I_{\mathrm{BIAS}}=I_{\mathrm{TH}}, I_{\mathrm{AMP}}=4 I_{\mathrm{TH}}$ and $f_{\mathrm{GS}}=1 \mathrm{GHz}$ : (a) Temporal intensity (solid line, left axis) and instantaneous frequency (open circles, right axis) and (b) spectral intensity (solid line, left axis) and group delay (open circles, right axis). 
pulse shown in Fig. 1 and varying the interferometer FSR and ER, considering that $\mathrm{FSR}=1 / \tau$ and $\mathrm{ER}=10 \log _{10}\left(P_{\mathrm{MAX}} / P_{\mathrm{MIN}}\right)$, where $P_{\mathrm{MAX}}$ and $P_{\mathrm{MIN}}$ are the maximum and minimum transmission of the TF of the interferometer, respectively.

In a first set of simulations the ER was changed and the FSR was fixed at a value equal to the pulse bandwidth at $1 / e^{2}, 33.5 \mathrm{GHz}$. A minimum of the interferometer TF was placed at the pulse spectrum center $(f=0 \mathrm{GHz}$ in Fig. 1). The ER was varied by changing $k_{2}$, with $k_{1}=0.5$.

The calculated FTFF is shown in Fig. 3, for ER between $0 \mathrm{~dB}$ $\left(k_{2}=0\right.$, plain $\left.\mathrm{TF}\right)$ and $9.5 \mathrm{~dB}\left(k_{2}=0.2\right)$, while Fig. $4(\mathrm{a})$ shows the intensity and instantaneous frequency for three particular values of ERs $(0 \mathrm{~dB}, 3 \mathrm{~dB}$ and $9.5 \mathrm{~dB})$.

For $E R=0 \mathrm{~dB}$, a value of $\mathrm{FTFF}=0.48$ is obtained corresponding to the FTFF of the input pulse. As the ER is increased, the pulse peak is progressively flattened and the FTFF increases up to a maximum of 0.56 , obtained for $\mathrm{ER}=3 \mathrm{~dB}\left(k_{2}=0.025\right)$. This case is illustrated in Fig. 4(a). For greater values of the ER, the FTFF decreases, as the spectral dip in the interferometer TF is more pronounced and double peaked pulses are generated at the interferometer output, as shown in Fig. 4(a), for $E R=9.5 \mathrm{~dB}$.

Fig. 4(a) also shows that the instantaneous frequency variation of the original pulse (about $30 \mathrm{GHz}$ ) is maintained. For small values of the ER, the spectral phase profile of $H(f)$ presents a smooth phase transition, as shown in Fig. 4(b), where a maximum phase variation of 0.3 rads is observed. For higher values of ER, the phase jump is stepper, until reaching a $\pi$ transition for the maximum ER $\left(k_{2}=0.5\right)$, as confirmed by simulations. In Fig. 4 (c) the intensity and group delay of the output pulse spectrum are plotted. It is worth noting that the group delay of the output pulse is almost the same than the input pulse (Fig. 1), showing that the

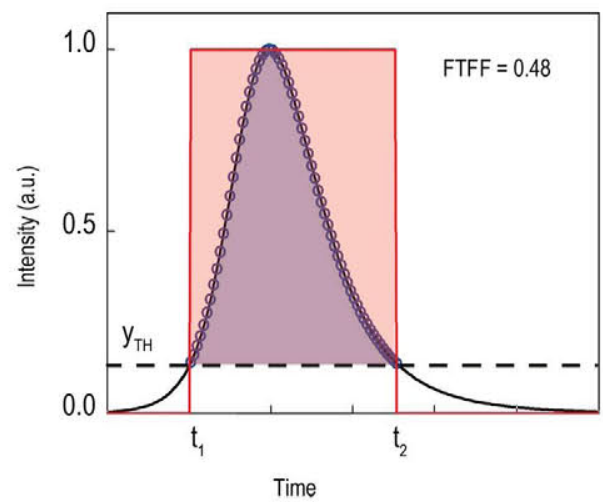

Fig. 2. Visual representation of the FTFF definition applied to the simulated input pulse, shown in Fig. 1. An ideal rectangular pulse is constructed from $y_{\mathrm{TH}}$ and the FTFF is given by the ratio between the areas under the rectangular pulse (red line) and $y(t)$ (open circles). (For interpretation of the references to color in this figure legend, the reader is referred to the web version of this article.)

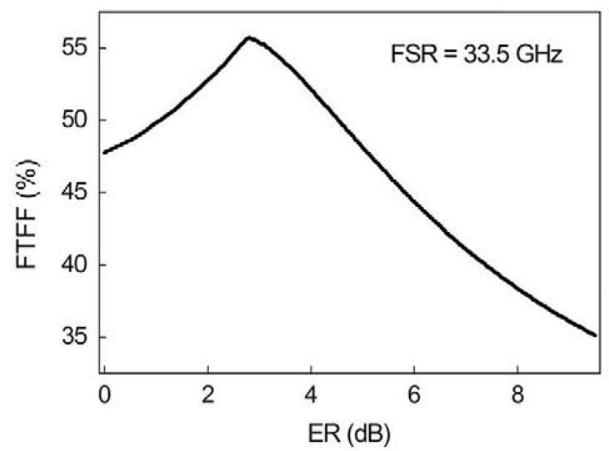

Fig. 3. The FTFF calculated for FSR $=33.5 \mathrm{GHz}$ as a function of the ER. temporal distribution of the pulse spectral content is maintained after passing through the interferometer.

A second set of simulations was performed with fixed $E R=3 \mathrm{~dB}$ and varying the FSR. A minimum of the interferometer TF was always considered at the center of the input pulse spectrum. The calculated FTFF as a function of the FSR and the pulse intensity and instantaneous frequency for selected examples are shown in Figs. 5 and 6 , respectively.

Maximum values ( $\sim 0.57$ ) of the FTFF parameter are found for a FSR between $25 \mathrm{GHz}$ and $30 \mathrm{GHz}$. The intensity and instantaneous frequency obtained for FSR $=28 \mathrm{GHz}$ are shown in Fig. 6: a flat top pulse with slightly more pronounced oscillations of the peak plateau, with respect to the case of $F S R=33.5 \mathrm{GHz}$, is obtained and the same total amount of instantaneous frequency variation of the input pulse is maintained.

For smaller values of the FSR, more than one spectral period of $H(f)$ falls into the pulse spectrum, thus exiting the regime of operation, where a notch-like TF has been assumed as the required filter for flat top pulse generation. As an example, the case $\mathrm{FSR}=10 \mathrm{GHz}$ is shown in Fig. 6, where a satellite pulse preceding the main pulse peak and an irregular and discontinuous profile of the instantaneous frequency can be observed. For FSR $\gtrsim 30 \mathrm{GHz}$, the spectral dip in $H(f)$ exceeds the pulse spectral bandwidth, broadening the pulse duration and smoothing the pulse peak plateau, as shown in Fig. 6, for the case FSR $=60 \mathrm{GHz}$.

The result obtained in Fig. 5 is understood as a simple filter design rule, following which the optimal value for FSR is slightly less than the full pulse spectrum at $1 / e^{2}$.

Previous results define two simple conditions for filter design: (i) a minimum of transmission of $|H(f)|^{2}$ must be placed at the center spectrum and the FSR has to be in the order or slightly smaller than the full spectrum width (measured at $1 / e^{2}$ of the maximum), (ii) small values of the ER ( $3 \mathrm{~dB}$ for the input pulse considered in the simulations) are the optimal choice and can be obtained with $k_{1}=0.5$ and varying $k_{2}$ until a flat top pulse profile is obtained.

Concerning the spectral phase of the proposed filter, an ideal constant phase profile has been assumed describing the proposed principle of operation, which is not the case for a real interferometer TF. However, simulations have shown that, for the cases of interests, the instantaneous frequency of the output and input pulse are similar. This is due to the fact that for the generation of a flat top pulse profile a small ER is required, which corresponds to smooth spectral phase transitions of $H(f)$, as predicted by theory and pointed out in detail in Ref. [12]. In fact, a spectral phase $\pi$ jump at a minimum transmission frequency is expected only in case of a 50:50 interferometer, i.e. with maximum ER. For smaller values of ER the spectral phase transition is smoother and its effect is negligible, as previously shown with presented simulation results.

The proposed technique implies pulse energy loss, as part of the input pulse spectrum is suppressed for shaping the pulse intensity profile. On the other hand, the small ER required for flat top pulse generation causes a total energy loss of 53\%, as obtained from simulation, by comparing the spectral energy of the output and input pulses, in the cases of flat top pulse generation, i.e. $\mathrm{FSR}=28 \mathrm{GHz}$ and $33.5 \mathrm{GHz}$ and $\mathrm{ER}=3 \mathrm{~dB}$ (see Figs. 4 and 6 ).

\section{Experiments}

The experimental set-up is shown in Fig. 7. The output of a GS Laser Diode (GS LD) is split in two paths via a 50:50 fiber coupler: one path is directly connected to a fast (20 GHz bandwidth) photodiode (PD) and to the channel $1(\mathrm{CH} 1)$ of a digital sampling 
a

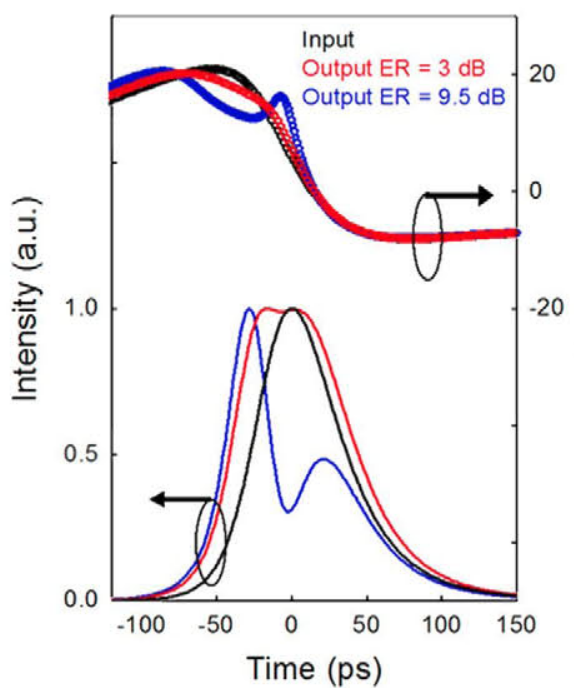

b

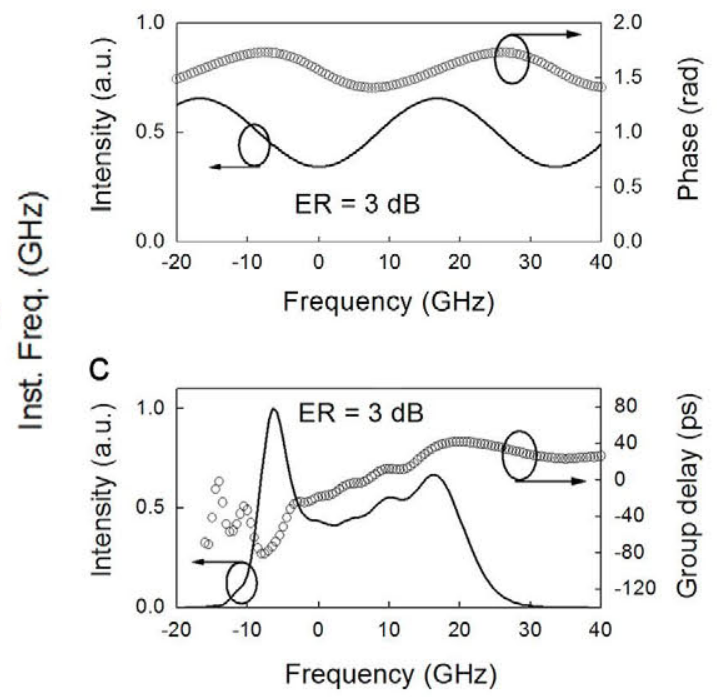

Fig. 4. Simulations results: (a) intensity (left axis) and instantaneous frequency (right axis) profiles of the input pulse (black) and of the pulses obtained with FSR $=33.5 \mathrm{GHz}$ and $E R=3 \mathrm{~dB}$ (red) and $E R=9.5 \mathrm{~dB}$ (blue). For the case $E R=3 \mathrm{~dB}$ : (b) interferometer TF: intensity (solid line, left axis) and phase (open circles, right axis), and (c) output spectrum: intensity (solid line, left axis) and group delay (open circles, right axis). (For interpretation of the references to color in this figure legend, the reader is referred to the web version of this article.)

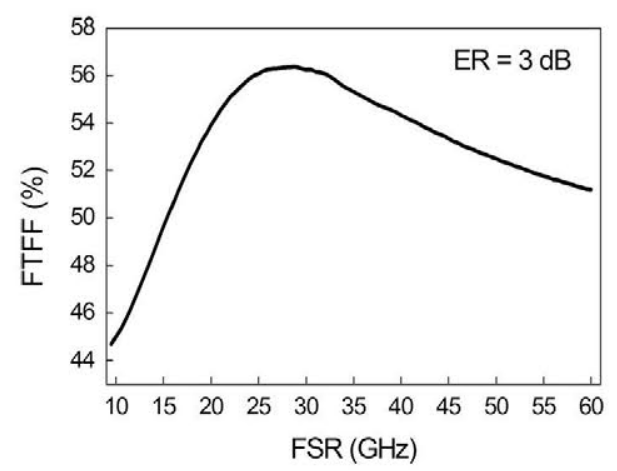

Fig. 5. The FTFF calculated as a function of the FSR, for $E R=3 \mathrm{~dB}$.

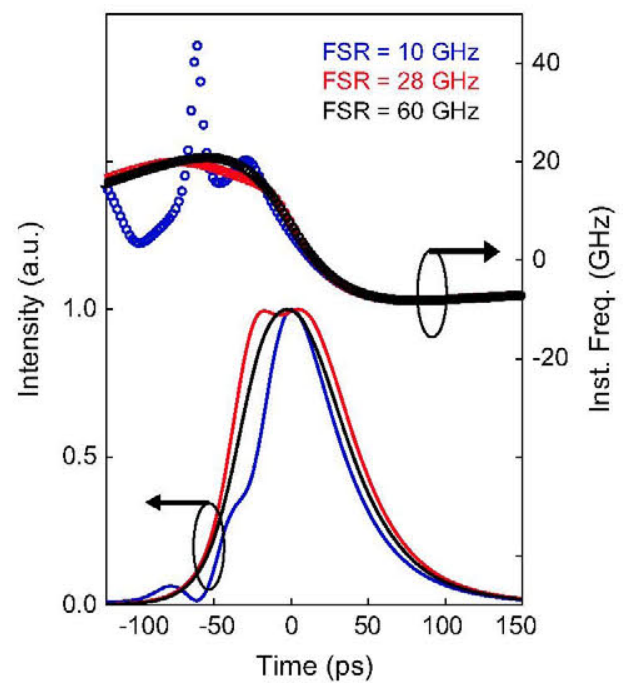

Fig. 6. Simulations results: intensity (solid lines, left axis) and instantaneous frequency (open circles, right axis) profiles of the pulses obtained with $E R=3 \mathrm{~dB}$ and $\mathrm{FSR}=10 \mathrm{GHz}$ (blue), $\mathrm{FSR}=28 \mathrm{GHz}$ (red) and $\mathrm{FSR}=60 \mathrm{GHz}$ (blue). (For interpretation of the references to color in this figure legend, the reader is referred to the web version of this article.) oscilloscope (OSC, Tektronix DSA8000) and the other path is sent to the interferometer.

A polarization controller (PC, Newport) is used to align the linear polarization direction of the GS LD to the polarization axis of the linear polarizer $(P 1)$ at the interferometer input. The linear polarizer $P 1$ and an Erbium Doped Fiber Amplifier (EDFA) are used for measuring the interferometer TF when the LD is off and the Amplified Spontaneous Emission (ASE) spectrum of the EDFA is sent to the interferometer. Spectral measurements are performed with an Optical Spectrum Analyzer (OSA, Ando AQ6315B) with a FWHM bandwidth of $0.05 \mathrm{~nm}(6.25 \mathrm{GHz})$, not shown in Fig. 7.

The proposed MZ polarization based interferometer consists of a first Polarization Beam Splitter (PBS), two optical paths with variable temporal difference $\tau$, a second PBS and a Rotatable Linear Polarizer (RLP). A Half Wave Plate (HWP) is used for rotating the linear polarization orientation of the input light with respect to the first PBS axes. Varying the angle $\alpha$, corresponding to the angle difference between the polarization orientation after the HWP and the first PBS axes, allows changing the ratio of the light sent along the two interferometer arms, i.e. varying the coupling factor as $k_{1}=\cos ^{2}(\alpha)$.

Light travelling along the two interferometer arms with orthogonal polarization directions interfere at the RLP. The angle $\beta$ at which the polarization axis of the RLP is oriented with respect to the second PBS axes determines the ratio of the light coming from the two interferometer arms interfering at the RLP, thus defining the coupling factor $k_{2}$ as $\cos ^{2}(\beta)$. The RLP is a commercially available component (Rotatable Linear Polarizer, OZ Optics) with a resolution of $2^{\circ}$.

The two PBSs and the motorized translation stage are fiber coupled and sealed into a small $(105 \times 51 \times 25 \mathrm{~mm})$ metallic box (Differential Polarization Delay Line, DPDL, OZ Optics). The translation stage is computer controlled and the delay $\tau$ can be varied with a nominal resolution of $\Delta \tau=1.65 \mathrm{fs}$.

For flat top pulse generation, the central wavelength of the filter is set after calculation of the delay $\tau$, which defines the wavelength location of maxima and minima of the interferometer TF. This is obtained by imposing that two adjacent maxima of the TF occur at $\lambda_{1}$ and $\lambda_{2}$, where $\lambda_{1}$ and $\lambda_{2}$ are the wavelengths at which the input pulse spectrum is $1 / e^{2}$ of its maximum. In this 


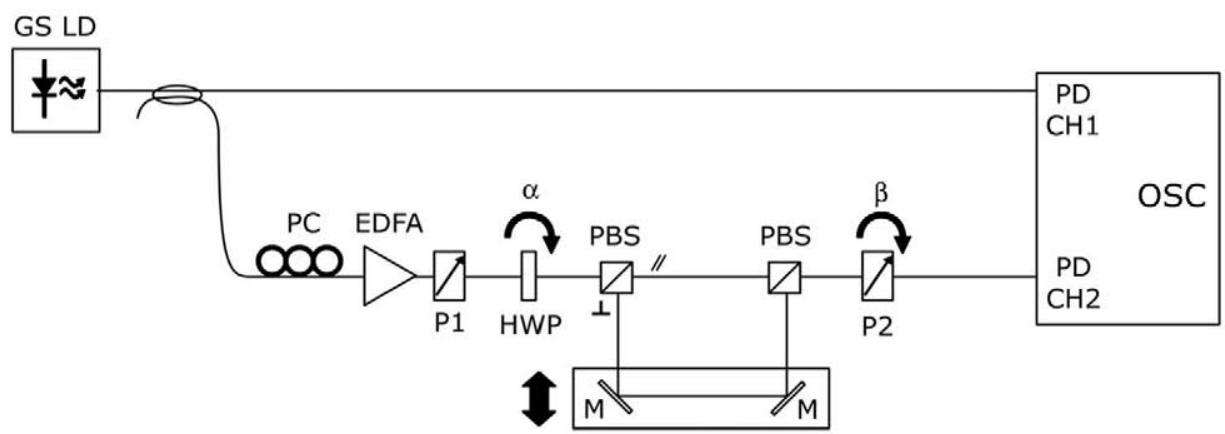

Fig. 7. Experimental set-up. Details are given in the text.
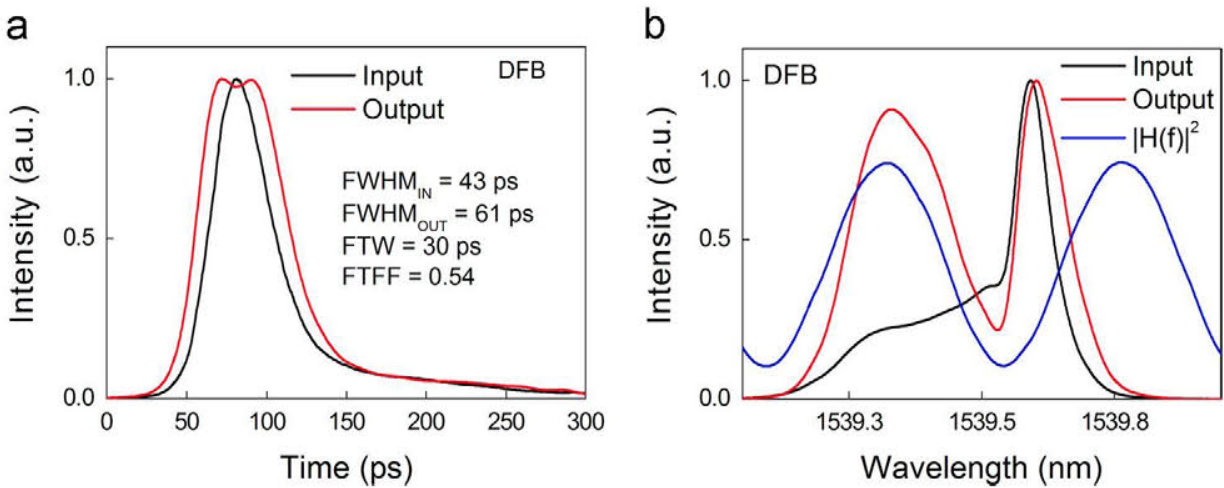

Fig. 8. Experimental results with the DFB laser, for $f_{\mathrm{RF}}=1 \mathrm{GHz}, P_{\mathrm{RF}}=16 \mathrm{dBm}$ and $I_{\mathrm{BIAS}}=I_{\mathrm{TH}}$ : (a) temporal intensity profiles of the input (black line) and output (red line) pulses, (b) input pulse (black line), output pulse (red line) spectra and interferometer TF (blue line). (For interpretation of the references to color in this figure legend, the reader is referred to the web version of this article.)

way a minimum of transmission of $|H(f)|^{2}$ coincides with the central wavelength of the input spectrum at $\left(\lambda_{1}+\lambda_{2}\right) / 2$.

Experimentally, the minimum step of the motorized translation stage, $\Delta \tau$, limits the obtainable resolution in setting the FSR and so the central frequency of the filter, $\nu_{0}=m$. FSR, being $m$ an integer. The resolution in the FSR, $\Delta \nu$, can be estimated as $\Delta \nu \approx \Delta \tau / \tau^{2}$, yielding values of $\Delta \nu$ between $16 \mathrm{MHz}$ and $4 \mathrm{MHz}$, for delays between $10 \mathrm{ps}$ and $20 \mathrm{ps}$ (as in this work), respectively. The resolution in the central frequency $\Delta \nu_{0}$, at constant $m$, is then $m \cdot \Delta \nu$, ranging between $32 \mathrm{GHz}$ and $16 \mathrm{GHz}$, for the delays considered. This resolution does not allow fine tuning of $\nu_{0}$ for the pulse bandwidth considered in experiments $\left(\sim 50 \mathrm{GHz}\right.$ at $\left.1 / e^{2}\right)$.

However, as the value of $m$ is high (2000-4000), it is always possible to find an interferometer minimum of transmission close to the central frequency of the input pulse spectrum by changing the delay time and selecting a different value of the integer $m$. For an arbitrary change of the delay $\tau$, the distance between the laser central frequency and the interferometer minimum of transmission ranges between zero and FSR $/ 2$. It is then possible to tune $\nu_{0}$ after a few trials, in which the translation stage of the DPDL is moved a few steps forward and backward with respect to the desired delay, until the desired $\nu_{0}$ is obtained and a flat top profile is observed at the oscilloscope. In our experiments, we were able to select the desired $\nu_{0}$ with a resolution better than $3 \mathrm{GHz}$ after a small number $(<10)$ of attempts.

Pulses obtained at the interferometer output were measured with a $45 \mathrm{GHz}$ photodiode (PD New Focus 1014) at the channel 2 $(\mathrm{CH} 2)$ of the sampling oscilloscope.

Two commercially available semiconductor lasers emitting in the $1550 \mathrm{~nm}$ region were used for pulse generation: a DFB laser (Uniphase) and a VCSEL (Raycan). Lasers were driven in gain switching conditions by means of a Radio Frequency (RF) sinusoidal signal at frequency $f_{\mathrm{RF}}$, with nominal output power $P_{\mathrm{RF}}$ and a $\mathrm{DC}$ current term $I_{\text {BIAS. }}$ The laser temperature was controlled with a Thermo Electric Cooler (TEC) and all measurements are performed at $25^{\circ} \mathrm{C}$.

Fig. 8 shows an example of the generation of a flat top pulse from the GS DFB laser. The input pulse had a FWHM duration of $43 \mathrm{ps}$, spectral width at $1 / e^{2}$ of $0.45 \mathrm{~nm}(56.2 \mathrm{GHz})$, centered at $1539.46 \mathrm{~nm}$ and a FTFF $=0.43$. The interferometer parameters were finely tuned to optimize the flat top profile by observing the temporal shape of the output pulse. Best results were obtained with $\tau=17.94 \mathrm{ps}(\mathrm{FSR}=55.7 \mathrm{GHz})$ and $\mathrm{ER}=8.7 \mathrm{~dB}$.

Very stable behavior (over $30 \mathrm{~min}$ ) has been observed in the interferometer TF and the output pulse shape, provided that the two optical paths of the interferometer were thermally insulated from ambient temperature fluctuations. This has been obtained by covering the DPDL with a $1.5 \mathrm{~cm}$ thick layer of polyurethane.

The filtered pulses have been characterized by measuring their FWHM, FTFF and a Flat Top Width (FTW), defined at 95\% of the pulse maximum and only pulses with less than $5 \%$ dip in the flat peak region have been considered. Results are presented in Fig. 8 (a), where a flat top pulse with FWHM of 61 ps and a FTW of 30 ps is shown. The FTFF obtained in this case is 0.54 . Fig. 8 (b) shows the input and output pulse spectra and the measured interferometer TF. A minimum of transmission was placed at about $1539.5 \mathrm{~nm}$ and a double peaked profile of the output pulse spectrum was obtained.

Similar experiments were performed with the GS VCSEL, see Fig. 9(a) and (b). The obtained pulses had a FWHM of 69 ps and spectral width at $1 / e^{2}$ of $0.53 \mathrm{~nm}(66.2 \mathrm{GHz})$. The calculated FTFF is 0.5 in this case. The central wavelength of the VCSEL was at about $10 \mathrm{~nm}$ away $(1550.6 \mathrm{~nm})$ from the DFB emission, demonstrating the tunability of the proposed technique in terms of operating wavelengths. In this case, the delay $\tau$ was set to $12.684 \mathrm{ps}$ and the ER of the interferometer was $6.6 \mathrm{~dB}$. The resulting output pulse had a FWHM of $104 \mathrm{ps}$, a FTW of 52 ps and FTFF $=0.59$. The 

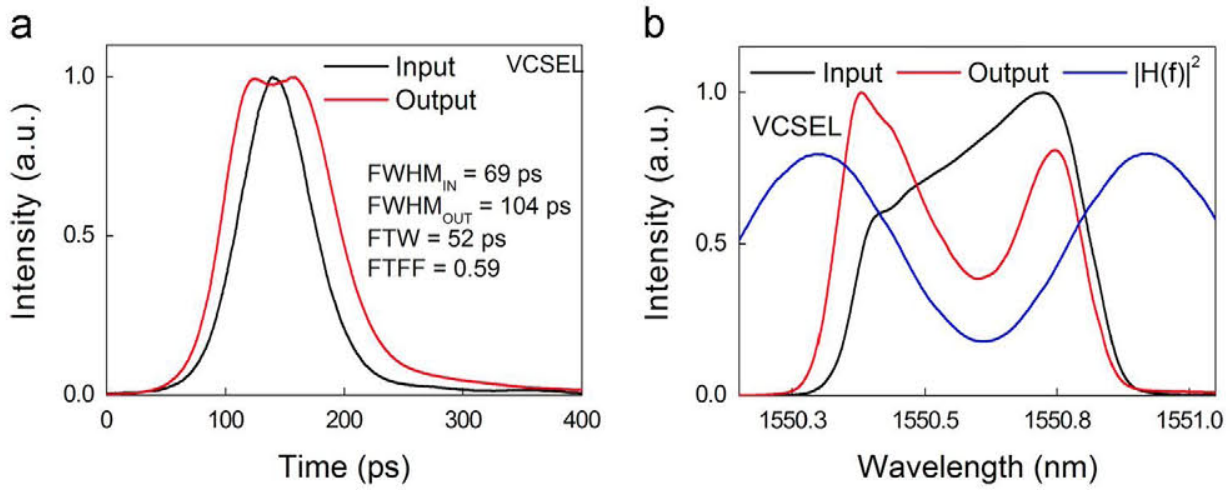

Fig. 9. Experimental results with the VCSEL, with $f_{\mathrm{RF}}=1 \mathrm{GHz}, P_{\mathrm{RF}}=14 \mathrm{dBm}$ and $I_{\mathrm{BIAS}}=1.1 I_{\mathrm{TH}}$ : (a) temporal intensity profiles of the input (black line) and output pulse (red line), (b) input pulse (black line), output pulse (red line) spectrum and interferometer TF (blue line). (For interpretation of the references to color in this figure legend, the reader is referred to the web version of this article.)
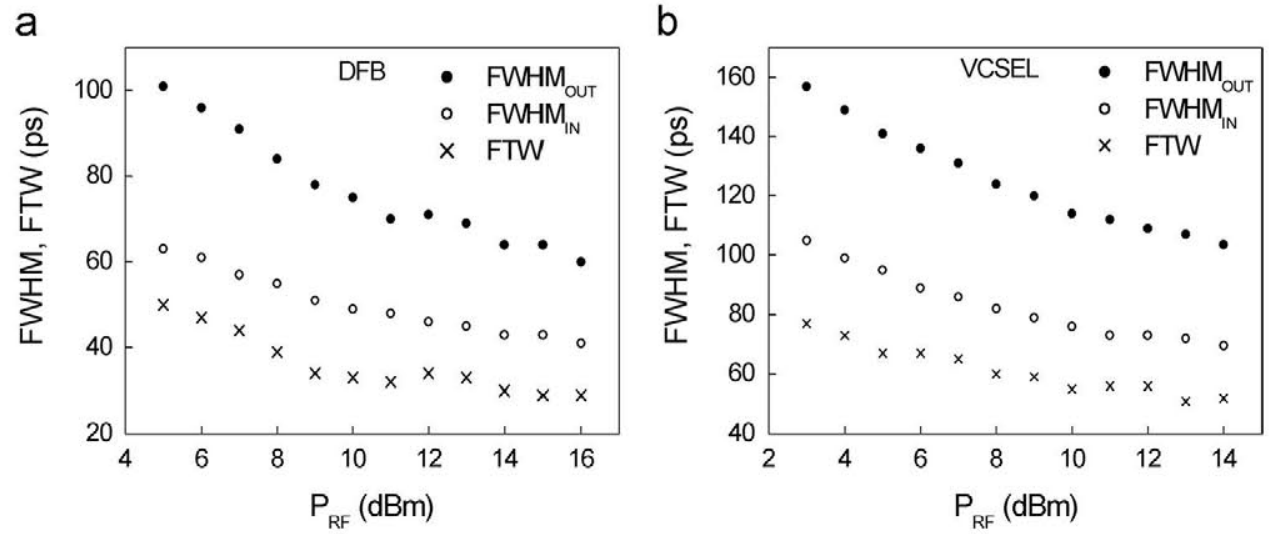

Fig. 10. Results obtained with DFB laser (a) and the VCSEL (b), as a function of $P_{\mathrm{RF}}$, with $f_{\mathrm{RF}}=1 \mathrm{GHz}$ and $I_{\mathrm{BIAS}}=I_{\mathrm{TH}}$ and $I_{\mathrm{BIAS}}=1.1 I_{\mathrm{TH}}$, for the DFB laser and the VCSEL, respectively. Input pulse FWHM (FWHM ${ }_{\mathrm{IN}}$, open circles), output pulse FWHM (FWHM $\mathrm{OuT}$, solid dots) and output pulse FTW (crosses).

spectral traces of the input and output pulses and the interferometer TF are shown in Fig. 9(b). Losses introduced by the spectral filtering process were experimentally determined by measuring the pulse peak amplitude after polarizer $P 1$ (input pulse) and after $P 2$ (output pulse) and by re moving the EDFA from the set-up. The peak power attenuation was about $67 \%$, for both the DFB laser and the VCSEL.

In order to test the flexibility of the proposed technique with respect to the input pulse durations, different input pulses were considered by varying the gain switching parameters for the device under study. The RF power was varied from $5 \mathrm{dBm}$ to $16 \mathrm{dBm}$ and from $3 \mathrm{dBm}$ to $14 \mathrm{dBm}$ for the DFB laser and the VCSEL, respectively. The interferometer TF was varied as described previously in order to obtain the best flat top pulse profiles at the interferometer output.

The results are shown in Fig. 10(a) and (b), for the DFB laser and the VCSEL, respectively. The pulse FWHM duration of a GS laser decreases with increasing $P_{\mathrm{RF}}$, until the second relaxation oscillation is excited and long tail or double peaked pulses are generated. The $P_{\mathrm{RF}}$ range has been chosen such that single peak pulses have been obtained for both devices. The FWHM of the generated pulses decreased with increasing $P_{\mathrm{RF}}$, from 63 ps to $41 \mathrm{ps}$ and from 105 ps to $69 \mathrm{ps}$, for the DFB laser and the VCSEL, respectively. The pulses obtained from the VCSEL show longer duration with respect to the DFB pulses, due to different device characteristics and operating conditions.

In all cases, flat top pulses with flat region oscillations $<5 \%$ were obtained, by placing a minimum of transmission close to the spectrum center and by varying the ER. The pulses obtained at the interferometer output had FWHM durations which are about $150 \%$ longer than the FWHM of the input pulses, for both devices and in all cases.

The FTW obtained at the interferometer output are about the $70 \%$ of the FWHM duration of the input pulses, ranging from $50 \mathrm{ps}$ to 29 ps in the DFB and from 77 ps to 52 ps in the VCSEL. The FTFF obtained in all experiments was around 0.55 and 0.59 for the DFB laser and the VCSEL, respectively. We attribute a greater value of the FTFF in the VCSEL to the different gain switching conditions and to the long tail of the pulses obtained with the DFB laser:

\section{Conclusions}

In this work, we propose a pulse shaping technique for the generation of flat top pulses from GS semiconductor lasers, using a polarization based Mach Zehnder interferometer with variable TF. The output pulse temporal profile is investigated theoretically as a function of the free spectral range and extinction ratio of the proposed interferometer, which are properly tuned by varying the two interferometer arms delay and the output coupling ratio, respectively.

The applicability of the proposed technique to different devices and pulses is demonstrated experimentally with the generation of flat top pulses from a GS DFB laser and a GS VCSEL, both emitting in the $1550 \mathrm{~nm}$ region. The gain-switching parameters are varied for each device under study in order to test the flexibility of the proposed technique on a wide range of input pulse durations: from $40 \mathrm{ps}$ (shortest FWHM from the GS DFB laser) to $100 \mathrm{ps}$ (longest FWHM from the GS VCSEL). 
Flat top pulses are obtained with FWHM about $150 \%$ of the input pulse FWHM and with intensity oscillations in the flat top region smaller than $5 \%$ of the pulse peak. Power losses, intrinsic to the proposed amplitude spectral filtering technique have been measured, giving an attenuation of $67 \%$ of the input pulse intensity peak.

\section{Acknowledgements}

The authors gratefully acknowledge the support of Ministerio de Economía y Competitividad of Spain under project TEC201238864-C03-02.

\section{References}

[1] P.P. Vasil'ev, I.H. White, J. Gowar, Rep. Prog. Phys. 63 (2000) 1997.

[2] R. Slavik, L.K. Oxenlowe, M. Galili, H.C.H. Mulvad, Y. Park, J. Azana, P. Jeppesen, IEEE Photonics Technol. Lett. 19 (2007) 1855.
3] C. Vicario, A. Trisorio, G. Arisholm, C.P. Haur, Opt. Lett. 37 (2012) 1619

[4] R. Selm, G. Krauss, A. Leitenstorfer, A. Zumbusch, Opt. Express 20 (2012) 5955.

[5] A.M. Weiner, Opt. Commun. 284 (2011) 3669.

[6] C. Guo, M. Nix, S.S. Yam, S. He, J. Lightwave Technol. 28 (2010) 876.

[7] Z. Tian, D.V. Plant, Opt. Lett. 36 (2011) 4542.

[8] Y. Park, M.H. Ashgari, T.-J. Ahn, J. Azaña, Opt. Express 15 (2007) 9584.

[9] P. Petropoulos, M. Ibsen, A.D. Ellis, D.J. Richardson, J. Lightwave Technol. 19 (2001) 746

[10] M.A. Preciado, M.A. Muriel, Opt. Lett. 34 (2009) 752.

11] R. Slavik, Y. Park, J. Azaña, IEEE Photonics Technol. Lett. 20 (2008) 806

12] R. Slavik, Y. Park, J. Azaña, Opt. Express 15 (2007) 6717.

[13] Y. Park, M. Kulishov, R. Slavík, J. Azaña, Opt. Express 14 (2006) 12670.

[14] A.M. Weiner, Rev. Sci. Instrum. 71 (2000) 1929.

[15] A. Consoli, I. Esquivias, Opt. Express 20 (2012) 22481

16] J.C. Cartledge, R.C. Srinivasan, J. Lightwave Technol. 15 (1997) 852.

[17] R. Hui, M. O'Sullivan, Fiber Optic Measurement Techniques, Elsevier Academic Press, Boston (2009) 2009129-258. 